\title{
MINIMUM FRICTION VELOCITY AND HEAT TRANSFER IN THE ROUGH SURFACE LAYER OF A CONVECTIVE BOUNDAR Y LA Y ER
}

\author{
ULRICH SCHUMANN \\ DFVLR, Institute of Atmospheric Physics, D-8031 Oberpfaffenhofen, Fed. Rep. Germany
}

(Received in final form 3 December, 1987)

\begin{abstract}
A simple model is deduced for the surface layer of a convective boundary layer for zero mean wind velocity over homogeneous rough ground. The model assumes large-scale convective circulation driven by surface heat flux with a flow pattern as it would be obtained by conditional ensemble averages. The surface layer is defined here such that in this layer horizontal motions dominate relative to vertical components. The model is derived from momentum and heat balances for the surface layer together with closures based on the Monin-Obukhov theory. The motion in the surface layer is driven by horizontal gradients of hydrostatic pressure. The balances account for turbulent fluxes at the surface and fluxes by convective motions to the mixed layer. The latter are the dominant ones. The model contains effectively two empirical coefficients which are determined such that the model's predictions agree with previous experimental results for the horizontal turbulent velocity fluctuations and the temperature fluctuations. The model quantitatively predicts the decrease of the minimum friction velocity and the increase of the temperature difference between the mixed layer and the ground with increasing values of the boundary layer/roughness height ratio. The heat transfer relationship can be expressed in terms of the common Nusselt and Rayleigh numbers, $\mathrm{Nu}$ and $\mathrm{Ra}$, as $\mathrm{Nu} \sim \mathrm{Ra}^{1 / 2}$. Previous results of the form $\mathrm{Nu} \sim \mathbf{R a}^{1 / 3}$ are shown to be restricted to Rayleighnumbers less than a certain value which depends on the boundary layer/roughness height ratio.
\end{abstract}

\section{Introduction}

The convective boundary layer (CBL) extends from the ground to the base of an elevated stable layer and is characterized by strong vertical mixing driven by upward heat flux from the surface. As summarized by Wyngaard (1985), the CBL is composed of three layers: the surface layer of height $h$, the mixed layer at height $z$ approximately between $h \leq z \leq 0.9 \mathrm{H}$, and the interfacial layer enclosing the temperature inversion at height $H$. The purpose of this paper is to investigate the surface layer for vanishing mean wind over homogeneous rough ground. We assume that the roughness can be characterized by a constant surface roughness height $z_{0}$. The height $h$ of the surface layer is usually defined such that in this layer the vertical fluxes vary by a small fraction, say $10 \%$. Unfortunately, this would imply different values of $h$ for the various fluxes. With respect to the vertical heat flux, $h / H \cong 0.1$. For momentum flux, $h$ is smaller or even undefined if the mean wind vanishes. Therefore, we define the surface layer as that layer where horizontal velocity fluctuations are large in comparison to vertical velucity fluctuations.

Turbulence in the mixed layer is driven by buoyancy. As shown by Deardorff

Boundary-Layer Meteorology 44 (1988) 311-326.

(C) 1988 by Kluwer Academic Publishers. 
(1970) and Wyngaard et al. (1971), the relevant convective velocity and temperature scales for the mixed layer are

$$
w_{*}=\left(\frac{g}{T} H Q_{s}\right)^{1 / 3}, \quad T_{*}=\frac{Q_{s}}{w_{*}},
$$

where $g$ is the gravitational acceleration, $T$ is the temperature $(1 / T$ represents the volumetric expansion coefficient) and $Q_{s}$ denotes the surface 'temperature flux', $Q_{s}=\overline{\left(w^{\prime} T^{\prime}\right)_{s}}$. The over-bar denotes the time or ensemble mean value. The suitability of these scales implies that the mixed layer is only weakly sensitive to surface roughness and molecular diffusivities.

If the mean horizontal wind speed $\bar{u}(z)$ is nonzero, the surface layer can be described using the Monin-Obukhov (M-O) similarity theory (e.g., see Dyer, 1974). This theory relates the mean wind and mean temperature profiles to the surface friction velocity and the corresponding friction temperature scales:

$$
u_{*}=\left(\frac{-\bar{\tau}_{s}}{\rho}\right)^{1 / 2}, \theta_{*}=-\frac{Q_{s}}{u_{*}},
$$

where $\bar{\tau}_{s} / \rho=\overline{\left(w^{\prime} u^{\prime}\right)_{s}}$ is the turbulent momentum flux close to the surface.

As has been recognized, e.g., by Businger (1973a, b), the M-O-theory breaks down in the windless case. We cite Businger (1973b), p. 81, who argues "that when $\bar{u}=0$ and consequently $u_{*}=0$, there are still substantial horizontal motions near the surface which have been introduced by the large-scale convection". He assumes that the convection causes a local wind profile near the surface which achieves steady state at a time scale much smaller than the time scale $H / w_{*}$ of the convective circulation. Businger conjectures that "the structure of the profile must be approximately the same as in the case when there is large-scale mean wind. Consequently, there is locally shear production of turbulence, and locally a friction velocity" $\tilde{u}_{*}$ may be defined. Businger postulates that this minimum friction velocity is related to the scales of the CBL and the surface roughness height $z_{0}$ according to

$$
\frac{\tilde{u}_{*}}{w_{*}}=f\left(\frac{H}{z_{0}}\right),
$$

where $f\left(H / z_{0}\right)$ is a not yet specified function which decreases with increasing $H / z_{0}$.

Even though cases with vanishing mean wind velocity are rare in reality, they require proper parameterizations for atmospheric circulation models to avoid singular results at positions where the mean wind ceases. Deardorff (1972) recommends the use of $u^{\prime}$, the root-mcan-square value of the horizontal velocity fluctuation, instead of $\bar{u}$ if $|\bar{u}|<u^{\prime}$ to compute $\tilde{u}_{*}$ from the M-O-equations. The present paper supports this recommendation on the basis of a simple model.

The knowledge of the function $f\left(H / z_{0}\right)$ is necessary, moreover, to determine the difference $\Delta \theta=\bar{\theta}_{s}-\bar{\theta}_{m}$ between the mean temperature of the surface and of 
the mixed layer because this difference depends on $\tilde{u}_{*}$. The temperature difference drives the heat flux in the CBL and is therefore of great importance (Tennekes, 1973, p. 205). On the other hand, it should be noted that the surface temperature is not very sharply defined for rough surfaces, difficult to measure and might be more of modelling importance than of real meaning. Here we assume that $\vec{\theta}_{s}$ represents the temperature at a height equal to the roughness height $z_{0}$. Panofsky and Dutton (1984), p. 147, suggest that the relevant surface roughness height for temperature is $\mu / u_{*}$, where $\mu$ is the molecular diffusivity of heat. Monin and Zilitinkevich (1969) distinguish between the temperature $\bar{\theta}_{s}=$ $\overline{\boldsymbol{\theta}}\left(z_{0}\right)$ and $\overline{\boldsymbol{\theta}}_{\mathrm{G}}$, the temperature at the ground, and propose the relation

$$
\Delta \theta_{G}=\bar{\theta}_{G}-\bar{\theta}_{s}=-0.13 \theta_{*}\left(\frac{u_{*} z_{0}}{\nu}\right)^{0.45}
$$

where $\nu$ is the kinematic viscosity.

The physical picture described by Businger (1973a, b) has been sketched already by Prandtl (1932). He and Priestley (1954) found $\Delta \theta \sim z^{-1 / 3}$ by considering the dynamics of buoyant elements which are mixing with the environment by turbulence on smaller scales. Monin and Yaglom (1971), p. 435, report on the results of several Soviet scientists and obtain the same dependence from the requirement that the $\mathrm{M}-\mathrm{O}$-theory gives a finite temperature difference for $\bar{u} \rightarrow 0$. They restrict the validity of this result for $z /|L| \gg 1$, where $L$ is the Obukhovlength (defined in the appendix). If one applies it nevertheless for $z=z_{0}$, it gives

$$
\Delta \theta=C Q_{s}^{2 / 3}\left(\frac{g}{T} z_{0}\right)^{-1 / 3}=C T *\left(\frac{H}{z_{0}}\right)^{1 / 3} .
$$

The magnitude of the coefficient $C$ is uncertain. Monin and Yaglom (1971), p. 499 , summarize several observations and deduce $2.3 \leq C \leq 3.6$. The resultant temperature difference $\Delta \theta$ is independent of molecular diffusivities.

As noted by Deardorff and Willis (1985), p. 210, "the problem of determining a simple, reliable parameterization of the surface heat flux under conditions of vanishingly small mean flow is still not satisfactorily solved". They repeat Deardorff's (1972) proposal and refer to Townsend (1964) who measured

$$
Q_{s}=C_{s}\left(\frac{g}{T} \frac{\mu^{2}}{\nu}\right)^{1 / 3}(\Delta \theta)^{4 / 3}
$$

for natural convection heat transfer over an ice surface. Deardorff and Willis (1985) found large scatter of the constant $C_{s}(0.10$ to 0.24$)$ and found that $C_{s}$ decreases in time with growing height of the CBL. This parameterization does not account for surface roughnesses and appears to be limited to very smooth surfaces therefore.

In the literature on heat transfer, it is common practice to express the relationship between the flux $Q_{s}$ and the effective temperature difference $\Delta \theta$ in 
terms of the Nusselt, Rayleigh, and Prandtl numbers,

$$
\mathrm{Nu}=\frac{Q_{s} H}{\mu \Delta \theta}, \quad \mathrm{Ra}=\frac{(g / T) \Delta \theta H^{3}}{\mu \nu}, \quad \operatorname{Pr}=\frac{\nu}{\mu} .
$$

With these numbers, Equation (5) becomes

$$
\mathrm{Nu}=C^{-3 / 2}\left(\frac{z_{0}}{H} \mathrm{Ra} \mathrm{Pr}\right)^{1 / 2},
$$

while Equation (6) can be written as

$$
\mathrm{Nu}=C_{s} \mathrm{Ra}^{1 / 3} \text {. }
$$

The difference in the values of the exponent of Ra suggests that Equations (8) and (9) describe different heat transfer processes. It is thus interesting to compare with the heat transfer relationship

$$
\begin{aligned}
\mathrm{Nu}=0.069 \mathrm{Ra}^{1 / 3} \mathrm{Pr}^{0.074} \text { for } \quad & 1.5 \cdot 10^{5} \leq \mathrm{Ra} \leq 6.8 \cdot 10^{8} \\
& 0.02 \leq \operatorname{Pr} \leq 8750,
\end{aligned}
$$

found by Globe and Dropkin (1959) for turbulent Rayleigh-Benard convection between two horizontal plane plates with gap $H$ heated uniformly from below. The measurements were obtained for $0.025 \mathrm{~m}<H<0.13 \mathrm{~m}$. This indicates that Equations $(6,10)$ describe the heat transfer over smooth surfaces while Equation (5) is designed for rough surfaces.

In this paper, a simple model is deduced which connects the physical picture envisaged by Businger (1973a, b) with a simple surfacc-layer model. As a result, the function $f\left(H / z_{0}\right)$ and a parameterization for $\Delta \theta$ will be determined. Moreover, we shall give an estimate of whether a surface of a convective boundary layer is to be considered as being smooth or rough.

\section{A Simple Model for the Surface Layer}

As sketched in Figure 1, the flow field in the turbulent CBL in the absence of mean wind is characterized by a circulation at scales of the order of the height $H$ of the CBL with high-speed rising motion in narrow thermals and low-speed sinking motion in the relativcly large surrounding area. Measurements of $\overline{w^{\prime 3}} / w_{*}^{3}$ (e.g., Caughey, 1982) and of the diameters of updrafts and downdrafts (e.g., Greenhut and Khalsa, 1987) show that this asymmetry exists. In the surface layer of thickness $h$, a horizontal motion with velocity $\bar{u}$ arises for continuity.

This picture would result from observations if we perform conditional sampling such that we obtain an average over an ensemble of thermals with suitably defined origins $\left(x_{i}, y_{i}\right), i=1,2, \ldots, n, n \gg 1$. If $f_{i}=f\left(x_{i}^{\prime}, y_{i}^{\prime}, z, t_{i}\right)$ is any component of the flow field of the $i$-th realisation of a convective cell, in which $x_{i}^{\prime}=x-x_{i}, y_{i}^{\prime}=y-y_{i}$ refer to the horizontal coordinates relative to the centre of the rising motion at time $t_{i}$, then the conditional ensemble average $\tilde{f}$ is the mean 


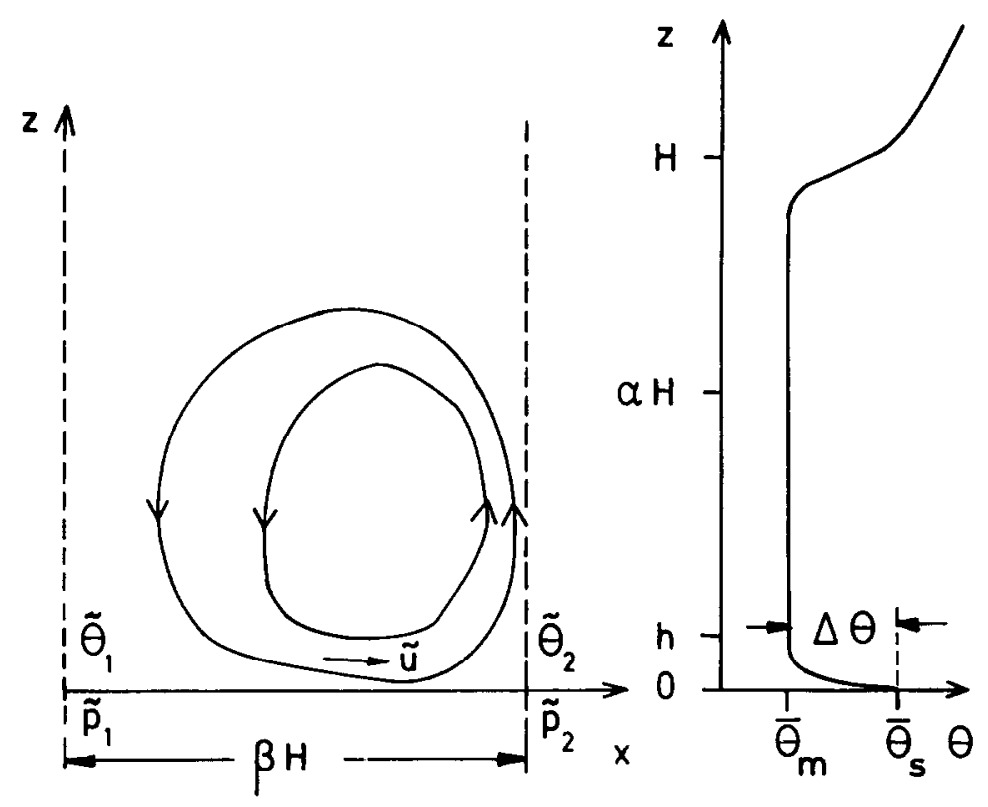

Fig. 1. Sketch of the circulation in the convective boundary layer (CBL) and the profile of the mean potential temperature $\bar{\theta}$ versus height $z$. The surface layer extends from the ground $(z=0)$ to the height $h$ and is topped by the mixed layer extending approximately up to height $H$.

value over all statistically independent fields $f_{i}$. Note that the resultant velocity $\tilde{u}$ is non-zero and space-dependent even if $\bar{u}$ is zero. Greenhut and Khalsa (1987) uscd similar definitions of conditional averages to evaluate the structure of a CBL.

In the surface layer, the fluid is heated from the surface by the constant temperature flux $\tilde{Q}_{s}=Q_{s}$. This causes the temperature in the surface layer to rise from $\tilde{\theta}_{1}$ at the foot of the sinking motion to $\tilde{\theta}_{2}$ at the foot of the rising thermal. The temperature variations cause buoyancy forces which result in hydrostatic pressure differences $\tilde{p}_{1}-\tilde{p}_{2}$. Moeng and Wyngaard (1986) have shown that about half the pressure fluctuations near the surface are caused by hydrostatic forces while the remainder is caused by turbulent interactions. It appears reasonable that the contribution of turbulent interactions are of smaller scale and contribute less to the horizontal pressure gradients. Moeng and Wyngaard (1986) have shown also that buoyancy-induced pressure fluctuations are small in the middle of the CBL. Thus we may estimate the horizontal pressure difference in the surface layer to be equal to

$$
\bar{p}_{1}-\tilde{p}_{2}=\rho \frac{g}{T} \alpha H\left(\tilde{\theta}_{2}-\tilde{\theta}_{1}\right) .
$$

Here, $\alpha H$ measures the height over which the thermals rise and sink with vertically constant temperatures $\theta_{2}$ and $\theta_{1}$, respectively; $\alpha$ is the first of a series of as yet undetermined model parameters which we shall introduce in this section 
and determine thereafter. The mean horizontal separation between the axis of rising and sinking motion is $\beta I I$ where $\beta$ is a further parameter of the present model.

In order to define heat and momentum balances, we consider a control volume of height $h$ for the surface layer together with heat and momentum fluxes as shown in Figure 2. This figure is consistent with the definition of the surface layer as that layer where horizontal motions dominate relative to vertical ones. To simplify the analysis, we assume that all parameters shown in Figure 2 are constants. For example, $\tilde{u}$ corresponds to the mean value of the conditional ensemble average of the horizontal velocity in the control volume. Moreover, we assume that the convective motion is close to steady state. As a consequence, the horizontal temperature difference $\tilde{\theta}_{2}-\tilde{\theta}_{1}$ is determined by the heat balance

$$
h \tilde{u}\left(\tilde{\theta}_{2}-\tilde{\theta}_{1}\right)=H \beta\left(Q_{s}-\tilde{Q}_{m}\right),
$$

where $\tilde{Q}_{m}$ is the temperature flux leaving the top of the surface layer by means of both fine-scale turbulence and convective-scale transport $\tilde{w} \tilde{\theta}$ averaged over the surface at $z=h$. The flux $\tilde{Q}_{m}$ is a certain fraction $\left(1-\gamma_{h}\right) Q_{s}$ of the surface flux. Again, $\gamma_{h}, 0<\gamma_{h}<1$, is a free model parameter. For small entrainment at the top of the CBL, $\gamma_{h} \cong h / H$ (Wyngaard, 1985). Thus

$$
h \tilde{u}\left(\tilde{\theta}_{2}-\tilde{\theta}_{1}\right)=H \beta \gamma_{h} Q_{s} .
$$

The horizontal momentum balance for steady state is

$$
\left(\tilde{p}_{1}-\tilde{p}_{2}\right) \boldsymbol{h}=\left(\tilde{\tau}_{m}-\tilde{\tau}_{s}\right) \boldsymbol{\beta} H,
$$

where the difference of the stresses at the interface to the mixed layer $\left(\tilde{\tau}_{m}\right)$ and at the bottom $\left(\tilde{\tau}_{s}\right)$ balances the pressure force. Note that these stresses are non-zero in the conditional ensemble average while the corresponding standard mean values $\bar{\tau}_{m}$ and $\bar{\tau}_{s}$ are zero.

Now, we relate the conditional ensemble averages to the standard mean values

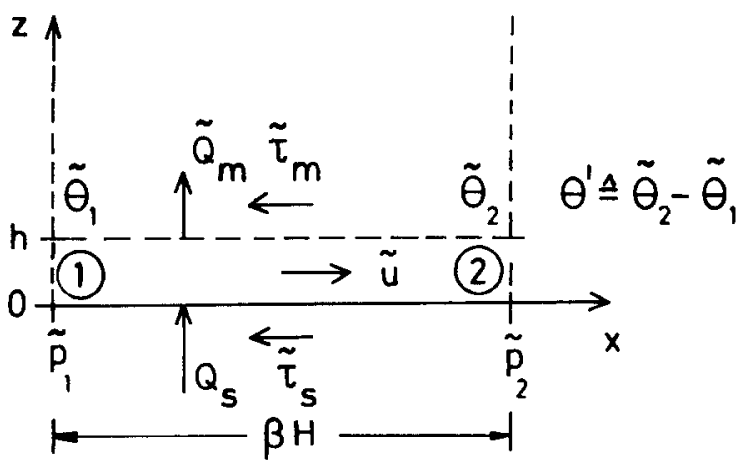

Fig. 2. Sketch of the control volume representing the surface layer of the CBL. 
(denoted by the over-bar) and the corresponding root-mean-square values (denoted by primes):

$$
\tilde{u} \cong u^{\prime}, \quad \tilde{\theta}_{2}-\bar{\theta}_{1} \cong \theta^{\prime}, \quad \frac{1}{2}\left(\tilde{\theta}_{1}+\tilde{\theta}_{2}\right)=\bar{\theta}_{m} .
$$

Here, the first two equations assume that the turbulent fluctuations are caused mainly by the convective circulation. The second equality is quantitatively supported by results of Greenhut and Khalsa (1987) who determined temperatures in updrafts and downdrafts by conditional sampling from aircraft measurements. The third equation describes a reasonable approximation because turbulent temperature fluctuations are small relative to the temperature difference $\Delta \theta=\bar{\theta}_{s}-\bar{\theta}_{m}$. One could have introduced further model coefficients of order unity to correct for these approximations but they would combine with the parameter $\beta$ in the subsequent analysis and are omitted therefore.

From Equations $(11,14)$, we may eliminate the pressure difference and obtain

$$
\tilde{\tau}_{m}-\tilde{\tau}_{s}=\alpha \gamma_{h} \rho \frac{g}{T} \frac{H Q_{s}}{u^{\prime}} .
$$

Using the definition for $w_{*}$, Equation (1), we find

$$
u^{\prime}\left(\tilde{\tau}_{m}-\tilde{\tau}_{s}\right)=\alpha \gamma_{h} \rho w_{*}^{3} .
$$

This together with Equations $(12,15)$ in reordered form

$$
\boldsymbol{\theta}^{\prime}=\boldsymbol{\beta} \gamma_{h} \frac{H}{h} \frac{Q_{s}}{u^{\prime}}
$$

is the result which we obtain from heat and momentum balances.

In order to close the system, we need parameterizations for the momentum fluxes $\tilde{\tau}_{s}$ and $\tilde{\tau}_{m}$, and the surface temperature flux $Q_{s}$ as a function of $u^{\prime}$ and $\Delta \theta$. At the surface, we assume that the $\mathrm{M}-\mathrm{O}$-theory may be applied to the conditional ensemble mean values of the convective motion although the theory presumes horizontal homogeneity and stationary flow with vertically constant heat and momentum fluxes. The flow is approximately stationary and homogeneous because $h$ is much smaller than $H$. The variation of the vertical heat flux will be also small because it varies approximately linearly with height. However, the momentum flux will show larger variations. Thus, the conditions of the $\mathrm{M}-\mathrm{O}$ theory are satisfied at best approximately. Therefore, in the absence of better proposals, we may apply the $\mathrm{M}-\mathrm{O}$-theory to obtain rough estimates. The $\mathrm{M}-\mathrm{O}$ equations in terms of the common mean values are summarized in the appendix. These equations predict velocity and temperature profiles which show little variations for $z>-L$, where $L$ is the Obukhov-length. Under the condition that this length scale is small in comparison to $h$, we obtain:

$$
\tilde{\tau}_{s}=-\rho \tilde{u}_{*}^{2},
$$




$$
u^{\prime}=\frac{\tilde{u}_{*}}{\kappa}\left[\ln \left(h / z_{0}\right)-\psi_{m}(h / \tilde{L})+\psi_{m}\left(z_{0} / \tilde{L}\right)\right] .
$$

Here $\kappa$ is the von Karman constant and $\tilde{L}$ the Obukhov-length related to the convective motion:

$$
\tilde{L}=-\frac{\tilde{u}_{*}^{3}}{\kappa Q_{s} g / T}=-\frac{H}{\kappa} \frac{\tilde{u}_{*}^{3}}{w_{*}^{3}} .
$$

Note that $\tilde{L} \neq 0$ while the standard Obukhov-length vanishes. Likewise, the surface temperature flux $Q_{s}$ is related to the temperature difference $\Delta \theta$ between the ground and the interior of the surface layer at $z \cong h$ by

$$
\begin{aligned}
\Delta \theta & =-\tilde{\theta}_{*} \frac{1}{\kappa}\left[\ln \left(h / z_{0}\right)-\psi_{h}(h / \tilde{L})+\psi_{h}\left(z_{0} / \tilde{L}\right)\right], \\
Q_{s} & =-\tilde{u}_{*} \tilde{\theta}_{*}=w_{*} T_{*} .
\end{aligned}
$$

The momentum flux $\tilde{\tau}_{m}$ is caused to a small fraction by small-scale turbulence but mainly by the convective motion $\rho \tilde{u}(x, y, z) \tilde{w}(x, y, z)$ averaged over the top surface of the surface layer at $z=h$. As Figure 1 suggests, sinking motion transports velocity with small horizontal velocity towards the centre of the thermal while rising motion near the thermal transports high-speed velocity upwards. This asymmetry causes $\tilde{\tau}_{m}$ to be positive on average and controlled by the convective velocity scale, i.e.,

$$
\tilde{\tau}_{m}=\gamma_{m} \rho w_{*}^{2} .
$$

Again, $\gamma_{m}$ is a free model parameter of order unity which depends on the value of $h / H$. It is reasonable to assume that the momentum flux by the convective circulation is much greater than the momentum flux at the surface, so that

$$
\left|\tilde{\tau}_{s}\right| \ll \tilde{\tau}_{m} .
$$

This is consistent with the already used assumption of $-h / \tilde{L} \gg 1$. In the following, we shall neglect the contribution of the surface stress relative to the stress at the interface to the mixed layer. This would not be necessary in principle but is justified for the given arguments and is technically necessary because otherwise the value of $u^{\prime}$ has to be determined from the roots of a cubical polynomial.

Now the system is closed and we may eliminate the momentum and heat fluxes from Equations $(17,18)$. Inserting Equations $(24,25)$ in (17) gives

$$
\frac{u^{\prime}}{w_{*}}=\frac{\alpha \gamma_{h}}{\gamma_{m}} .
$$

This together with Equation (20) results in

$$
\frac{\tilde{u}_{*}}{w_{*}}=\frac{a \gamma_{h}}{\gamma_{m}} \frac{\kappa}{\ln \left(h / z_{0}\right)-\psi_{m}(h / L)+\psi_{m}\left(z_{0} / \tilde{L}\right)}, \quad \frac{\tilde{\theta}_{*}}{T_{*}}=-\frac{w_{*}}{\tilde{u}_{*}} .
$$


Here we need to know $\tilde{L}$, which we obtain from Equations $(21,27)$ :

$$
\tilde{L}=-H\left(\frac{\alpha \gamma_{h}}{\gamma_{m}}\right)^{3} \frac{\kappa^{2}}{\left[\ln \left(h / z_{0}\right)-\psi_{m}(h / \tilde{L})+\psi_{m}\left(z_{0} / \tilde{L}\right)\right]^{3}}
$$

From Equations (22) and (27) follows

$$
\frac{\Delta \theta}{T_{*}}=\frac{\gamma_{m}}{\kappa^{2} \alpha \gamma_{h}}\left[\ln \left(\frac{h}{z_{0}}\right)-\psi_{m}\left(\frac{h}{\tilde{L}}\right)+\psi_{m}\left(\frac{z_{0}}{\tilde{L}}\right)\right]\left[\ln \left(\frac{h}{z_{0}}\right)-\psi_{h}\left(\frac{h}{\tilde{L}}\right)+\psi_{h}\left(\frac{z_{0}}{\tilde{L}}\right)\right] .
$$

Equation (18) combined with (26) becomes

$$
\frac{\theta^{\prime}}{T_{*}}=\frac{\beta \gamma_{m}}{\alpha} \frac{H}{h}
$$

Equation (27) defines the function $f\left(H / z_{0}\right)$. For evaluation, one has to compute $\tilde{L}$ by inverting Equation (28) for given value of $H / z_{0}$.

\section{Determination of the Model Parameters}

The thickness of the surface layer $h$ has been defined as that layer where horizontal motions dominate relative to vertical ones. A reasonable estimate is

$$
\frac{h}{H}=0.1 \text {. }
$$

This estimate is backed by measurements of the vertical and horizontal velocity fluctuations as depicted in Figure 4.18 of Caughey (1982). It is consistent with the value obtained from the condition that the vertical heat flux varies by less than $10 \%$ for small entrainment heat-flux at the top of the CBL (Wyngaard, 1985). In addition, the above theory contains effectively only two independent empirical coefficients (for given $\mathrm{M}$-O flux-profile relationships and given value of the von Karman constant $\kappa$ ), namely

$$
\frac{\alpha \gamma_{h}}{\gamma_{m}}=\frac{u^{\prime}}{w_{*}} \text { and } \quad \frac{\beta \gamma_{m}}{\alpha}=\frac{h}{H} \frac{\theta^{\prime}}{T_{*}} .
$$

These coefficients can be determined from experiments which apply for $-h / \tilde{L} \gg$ 1. We shall see that this is usually the case for experiments in atmospheric boundary layers with moderately large surface roughnesses and vanishing mean wind. A test is the independence of $u^{\prime} / w_{*}$ from $H / z_{0}$ and from the ratio $h / H$. The data for $u^{\prime} / w_{*}$ shown in Figure 4.18 of Caughey (1982) satisfy this condition. They imply

$$
\frac{\alpha \gamma_{h}}{\gamma_{m}}=0.7 \pm 0.1
$$

The result is supported also by the measurements of Deardorff and Willis (1985) 
in a laboratory convection experiment and by results of large eddy simulations as used by Deardorff (1972).

For the preselected value $h / H=0.1$, we obtain from the data for $\theta^{\prime} / T_{*}$ shown in Figure 4.19 of Caughey (1982):

$$
\frac{\beta \gamma_{m}}{\alpha}=0.3 \pm 0.05
$$

Alternatively, we might use the correlation

$$
\frac{\theta^{\prime}}{T_{*}}=1.8^{1 / 2}\left(\frac{H}{h}\right)^{1 / 3}
$$

given by Kaimal et al. (1976). It implies

$$
\frac{\beta \gamma_{m}}{\alpha}=1.8^{1 / 2}(h / H)^{2 / 3}=0.289 \text { for } \frac{h}{H}=0.1 .
$$

This result is consistent with Equation (34) and reflects moreover the fact that the coefficient $\gamma_{m}$, defined in Equation (24), will increase with $h / H$ because of increasing vertical momentum transport by the convective circulation.

Based on these results, all remaining quantities can be evaluated. Figures 3 and

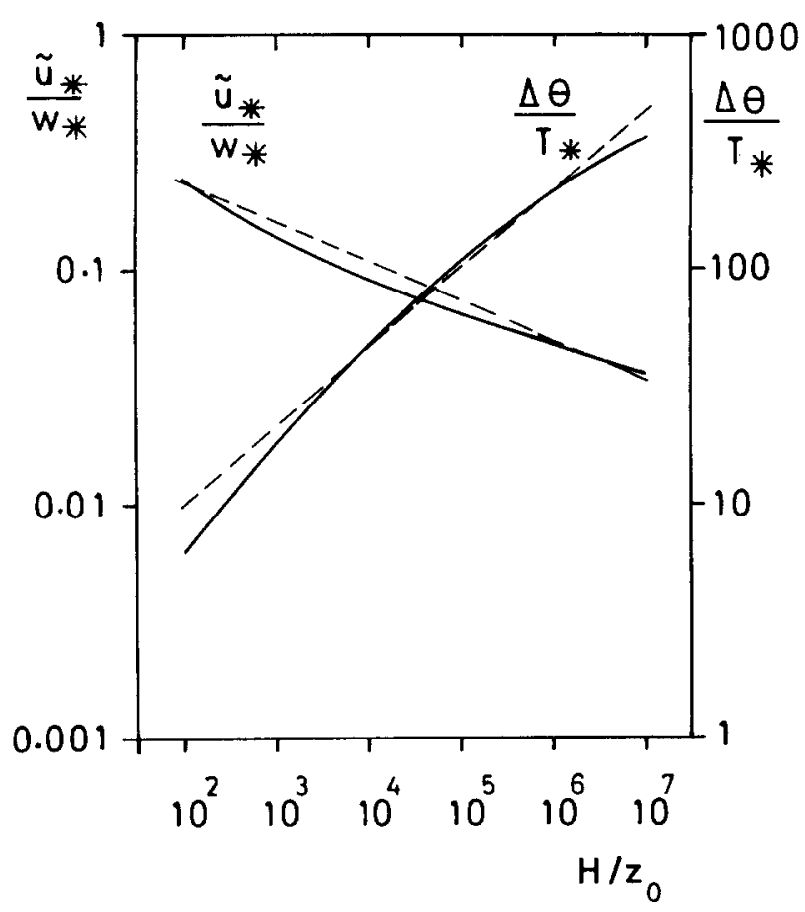

Fig. 3. Plot of minimum friction velocity $\tilde{u}_{*}$ over the convective velocity scale $w_{*}$ and of the temperature difference $\Delta \theta$ over the convective temperature scale $T_{*}$ versus the boundary layer/roughness height ratio $H / z_{0}$. The full curves correspond to Equations $(27,29)$, the dashed lines to Equations $(37,38)$. 


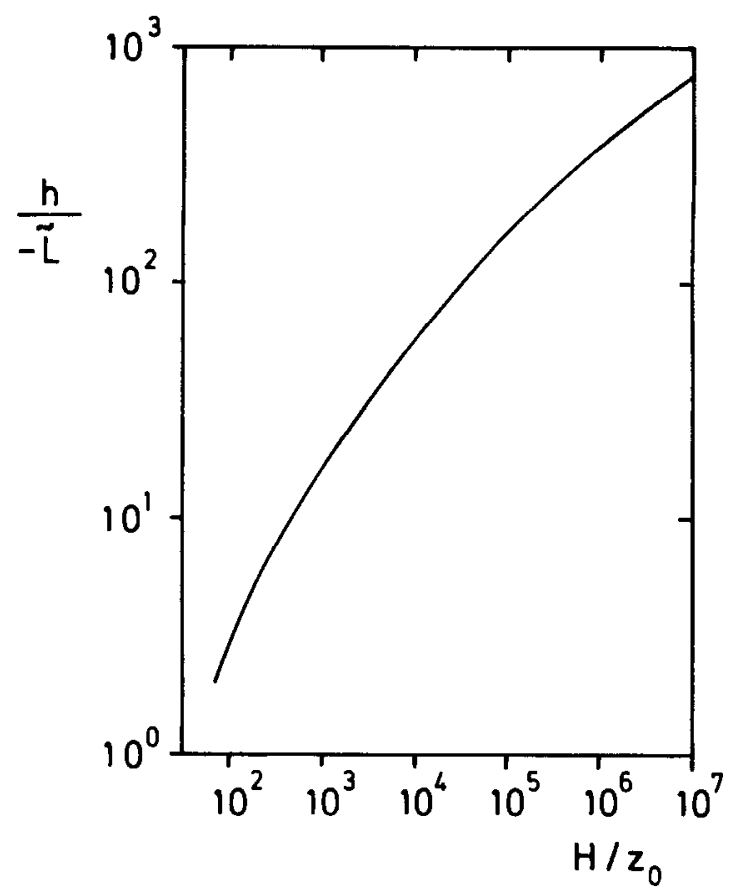

Fig. 4. Plot of the ratio of surface layer thickness $h$ over the Obukhov length for the convective motion $\tilde{L}$ versus the boundary layer/roughness height ratio $H / z_{0}$.

4 show the results for those quantities which are functions of $H / z_{0}$. The function $\tilde{u}_{*} / w_{*}$ versus $\left(H / z_{0}\right)$ is the function $f\left(H / z_{0}\right)$ postulated by Businger $(1973 \mathrm{a}, \mathrm{b})$. As predicted, it decreases with increasing $H / z_{0}$. The temperature difference $\Delta \theta / T_{*}$ strongly increases with $H / z_{0}$. For comparison it might be noted that Equation (6) would give a constant temperature difference. Figure 4 shows that $-h / \tilde{L}>1$ for $H / z_{0}>100$. This is the lower limit of the validity of the results because of the assumption expressed in Equation (25). Most mid-day atmospheric convective boundary layers satisfy this condition except for very large roughness heights like those for high trees or urban areas.

The dashed curves in Figure 3 correspond to

$$
\begin{aligned}
& \frac{\tilde{u}_{*}}{w_{*}}=0.52\left(\frac{H}{z_{0}}\right)^{-1 / 6}, \\
& \frac{\Delta \theta}{T_{*}}=\left(10 \frac{H}{z_{0}}\right)^{1 / 3} .
\end{aligned}
$$

The latter result corresponds to Equation (5) with $C=2.15$. This value is a little smaller than the estimates given by Monin and Yaglom (1971), p. 499. Obviously, these simple equations give good approximations to the Equations $(27,29)$ and may be of practical value, therefore. For example, by means of these 
correlations, Equation (4) can be rewritten as

$$
\frac{\Delta \theta_{G}}{T_{*}}=0.19\left(\frac{H}{z_{0}}\right)^{0.0917}\left(\frac{w_{*} z_{0}}{\nu}\right)^{0.45} \text {. }
$$

Typical values in an atmospheric CBL (Kaimal et al., 1976, run 6A1) are $w_{*}=2.4 \mathrm{~m} \mathrm{~s}^{-1}, \quad T_{*}=0.09 \mathrm{~K}, \quad H=2000 \mathrm{~m}, \quad z_{0}=0.02 \mathrm{~m}, \quad T=300 \mathrm{~K}, \quad \nu=$ $15 \cdot 10^{-6} \mathrm{~m}^{2} \mathrm{~s}^{-1}$. From these values, we obtain $H / z_{0}-10^{5}, u_{*}-0.15 \mathrm{~m} \mathrm{~s}^{-1}, u^{\prime}-$ $1.7 \mathrm{~m} \mathrm{~s}^{-1},-\tilde{L}=1.3 \mathrm{~m}, \theta^{\prime}=0.27 \mathrm{~K}, \Delta \theta=9.8 \mathrm{~K}$, and $\Delta \theta_{G}=1.9 \mathrm{~K}$. Kaimal et al. (1976) report minimum values of $u_{*}=0.18 \mathrm{~m} \mathrm{~s}^{-1}$ and of $-L=5.7 \mathrm{~m}$, but for non-zero mean wind. Thus the theory seems to predict realistic results.

\section{Heat Transfer Correlations and Limits of Validity}

If Equation (29) is expressed in terms of the characteristic numbers defined in (7), we obtain

$$
\mathrm{Nu}=F(\operatorname{RaPr})^{1 / 2}, F=\left(\frac{T_{*}}{\Delta \theta}\right)^{3 / 2}, \quad F \cong\left(\frac{z_{0}}{10 H}\right)^{1 / 2},
$$

where Equation (38) is used to obtain the approximation for $F=F\left(H / z_{0}\right)$. This result has been deduced for a rough surface. In order to be classified as rough, the surface roughness parameter $z_{0}$ has to be greater than the scale $\nu / \tilde{u}_{*}$ of the viscous layer. From the definitions of the various numbers, one easily obtains

$$
\frac{z_{0} \tilde{u}_{*}}{\nu}=\frac{\tilde{u}_{*}}{w_{*}}\left(\frac{T_{*}}{\Delta \theta}\right)^{1 / 2} \frac{z_{0}}{H}\left(\frac{\mathrm{Ra}}{\operatorname{Pr}}\right)^{1 / 2} .
$$

Using the approximate results, Equations $(37,38)$, give

$$
\frac{z_{0} \tilde{u}_{*}}{\nu}=0.354\left(\frac{z_{0}}{H}\right)^{4 / 3}\left(\frac{\mathrm{Ra}}{\operatorname{Pr}}\right)^{1 / 2}
$$

This number is greater than 10 , which might be a reasonable limit for a rough surface, if

$$
\operatorname{Ra}>560\left(\frac{H}{z_{0}}\right)^{8 / 3}, \quad \text { for } \operatorname{Pr}=0.7 .
$$

It requires, e.g., $\mathrm{Ra}>5.6 \cdot 10^{10}$ for $H / z_{0}=1000$, and $\mathrm{Ra}>5.6 \cdot 10^{18}$ for $\mathrm{H} / z_{0}=$ $10^{6}$. Since it is difficult to reach such high Rayleigh numbers in laboratory experiments, it can be surely assumed that Equation (10) has been obtained for effectively smooth surfaces.

For the parameter values given at the end of the previous section, we obtain $\mathrm{Ra}=8 \cdot 10^{18}, \mathrm{Nu}=2 \cdot 10^{6},\left(z_{0} \tilde{u}_{*} / \nu\right)=203$. Thus, the given example corresponds to a rough surface under pure convective conditions in spite of the not very large value of $z_{0}$. 


\section{Summary and Discussion}

The present model is based on the concept of conditional ensemble values of the structure of the CBL as shown in Figures 1 and 2 together with balances of heat and momentum in the surface layer and the Monin-Obukhov (M-O) theory for the heat and momentum transfer at the surface. From these considerations, explicit relationships have been deduced to determine the turbulent velocity fluctuations $\boldsymbol{u}^{\prime}$ and the turbulent temperature fluctuations $\boldsymbol{\theta}^{\prime}$ in the surface layer. The results are consistent with measurements summarized by Caughey (1982) and these data are used to determine the two free coefficients of the present model. From the model, we have determined the function $f\left(H / z_{0}\right)$ postulated by Businger $(1973 \mathrm{a}, \mathrm{b})$ and the temperature difference $\Delta \theta$ between ground and surface layer.

The largest uncertainty of the model originates presumably from application of the M-O-theory for conditions which do not fully satisfy the requirements of this theory. However, the sensitivity of the results to parameters of the M-Oequations is weak. For instance, replacing $h$ in Equations $(20,22)$ by $0.5 h$ causes $\tilde{u}_{*} / w_{*}$ to increase and $\Delta \theta / T_{*}$ to decrease at maximum by $(18.4,2.7,1.0) \%$ for $H / z_{0}=\left(10^{2}, 10^{4}, 10^{6}\right)$, respectively, and $H / z_{0}$ should be greater than $10^{3}$ for the present theory.

The results show that in common atmospheric cases the surface layer loses most of its circulation momentum to the mixed layer by asymmetrical large-scale convective motions while the momentum loss at the (rough) surface is negligible. This explains why the turbulence in the mixed layer is only weakly dependent on surface roughness. However, the shear-induced turbulence at the surface is responsible for the surface temperature flux relative to the temperature difference between ground and surface layer. In fact, this temperature difference drives the whole CBL since it controls the surface energy balance (including radiation) and thus the amount of sensiblc heat flux from the ground to the atmosphere. The value of $\tilde{u}_{*}$ is important to know for estimates of turbulent transports between the surface and the surface layer for vanishing mean wind velocity. The present theory could easily be extended to relate moisture differences with the latent heat flux or concentration differences with fluxes of other air constituents.

The heat transfer relationship obtained from the present model shows that the Nusselt number increases with the square root of the Rayleigh number whereas it increases with the third root of the Rayleigh number for smooth surfaces. Equation (43) specifies the limit between smooth and rough surfaces. Experiments are necessary to verify this result. It is reassuring that the result Equation (29) for $\Delta \theta / T_{*}$ is in close agreement with the prediction of Prandtl (1932) and others given by the dashed curve in Figure 3 and Equation (38).

No measurements have been found to check the validity of the predicted temperature difference $\Delta \theta$. This is not surprising in view of the difficulties in defining and measuring the surface temperature (together with $Q_{s}, z_{0}$, and $H$ ) 
but it is particularly unfortunate because such data would provide an independent check of Equation (29) since all model parameters in this equation have been fixed already. The large field experiments in 1968 in Kansas (Haugen et al., 1971) and 1973 in Minnesota (Kaimal et al., 1976) used temperature probes at towers with a minimum height position of $0.5 \mathrm{~m}$. This height is still much larger than $z_{0}$ in these experiments. Moreover, local measurements would probably not be representative for the surface temperature which drives the average heat flux over a larger surface area. Measurement of infrared radiation might give a suitable indicator of the temperature $\bar{\theta}_{G}$ at the ground which then can be related to the surface temperature $\bar{\theta}_{s}$ by Equation (39). Remote sensing of this radiation would also give the necessary average over a larger surface area.

Deardorff and Willis (1985) report that in their laboratory experiment $\Delta \theta$ was 'typically' $10 \mathrm{~K}$ while $T_{*}$ varies between 0.094 and $0.14 \mathrm{~K}$. In the present theory, see Figure 3 , this would imply $H / z_{0}$ varying between $3 \cdot 10^{4}$ and $10^{5}$. Unfortunately, Deardorff and Willis (1985) do not report values for $H / z_{0}$. However, for comparison such values would be of limited value because in this experiment the effective Rayleigh number is of the order $\mathrm{Ra}=10^{\circ}$ and thus not large enough in view of the criterium given in Equation (43).

We have started to apply large-eddy simulations for the CBI, to compute ensemble mean values of thermals and to verify the results given in Figure 3 . For this purpose, we are using the method described in Schumann et al. (1987). The results obtained corroborate the results reported in this paper. Details of these simulations are to be reported in Schmidt and Schumann (1989).

\section{Acknowledgments}

I thank Drs. J. A. Businger, T. Hauf, H. Kraus, F. T. M. Nieuwstadt, H. Schmidt and an anonymous reviewer for several hints and critical questions which helped me to improve the present paper.

\section{Appendix: Monin-Obukhov Relationships}

In Section 2, we apply the Monin-Obukhov (M-O) theory. The corresponding equations are well known but summarized here for completeness and to specify the values of the numerical parameters where we follow Paulson (1970) and Dyer (1974):

$$
\begin{aligned}
& \bar{u}(z)=\frac{u_{*}}{\kappa}\left[\ln \left(z / z_{0}\right)-\psi_{m}(z / L)+\psi_{m}\left(z_{0} / L\right)\right] \\
& \bar{\theta}(z)-\theta_{s}=\frac{\theta_{*}}{\kappa}\left[\ln \left(z / z_{0}\right)-\psi_{h}(z / L)+\psi_{h}\left(z_{0} / L\right)\right] \\
& u_{*}=\left(\frac{-\tau_{s}}{\rho}\right)^{1 / 2}, \quad \theta_{*}=-\frac{Q_{s}}{u_{*}}, \quad L=-\frac{u_{*}^{3}}{\kappa Q_{s} g / T}
\end{aligned}
$$




$$
\begin{aligned}
& \psi_{m}(\zeta)=2 \ln \left(\frac{1+\varphi_{m}^{-1}}{2}\right)+\ln \left(\frac{1+\varphi_{m}^{-2}}{2}\right)-2 \arctan \left(\varphi_{m}^{-1}\right)+\frac{\pi}{2} \\
& \psi_{h}(\zeta)=2 \ln \left(\frac{1+\varphi_{h}^{-1}}{2}\right) \\
& \varphi_{m}(\zeta)=(1-16 \zeta)^{-1 / 4}, \quad \varphi_{h}(\zeta)=(1-16 \zeta)^{-1 / 2}, \quad \kappa=0.41 .
\end{aligned}
$$

These equations apply for the unstable case, i.e., for $z / L \leq 0$, where the Obukhov-length $L$ is negative. For discussions of the values of the coefficients used, see Businger et al. (1971), Yaglom (1977), Viswanadham (1982), and Wier and Römer (1987). In a preliminary version of this paper, we had used the earlier proposals by Businger et al. (1971). The differences induced by this ambiguity are small. The terms $\psi_{m}\left(z_{0} / L\right)$ and $\psi_{h}\left(z_{0} / L\right)$ are negligible for $z_{0} / L \ll 1$.

\section{References}

Businger, J. A.: 1973a, 'A Note on Free Convection', Boundary-Layer Meteorol. 4, 323-326.

Businger, J. A.: 1973b, 'Turbulent Transfer in the Atmospheric Surface Layer', in D. A. Haugen (ed.), Workshop on Micrometeorology, Amer. Meteor. Soc., Boston, pp. 67-100.

Businger, J. A., Wyngaard, J. C., Izumi, Y., and Bradley, E. F.: 1971, 'Flux-Profile Relationships in the Atmospheric Surface Layer', J. Atmos. Sci. 28, 181-189.

Caughey, S. J.: 1982, 'Observed Characteristics of the Atmospheric Boundary Layer', in F. T. M. Nieuwstadt and H. van Dop (eds.), Atmospheric Turbulence and Air Pollution Modelling, D. Reidel Publ., Dordrecht, pp. 107-158.

Deardorff, J. W.: 1970, 'Convective Velocity and Temperature Scales for the Unstable Planetary Boundary Layer and for Rayleigh Convection', J. Atmos. Sci. 27, 1211-1213.

Deardorff, J. W.: 1972, 'Parameterization of the Planetary Boundary Layer for Use in General Circulation Models', Monthly Weather Rev. 100, 93-105.

Deardorff, J. W. and Willis, G. E.: 1985, 'Further Results from a Laboratory Model of the Convective Planetary Boundary Layer', Boundary-Layer Meteorol. 32, 205-236.

Dyer, A. J.: 1974, 'A Review of Flux-Profile Relationships', Boundary-Layer Meteorol. 7, 363-372.

Globe, S. and Dropkin, D.: 1959, 'Natural-Convection Heat Transfer in Liquids Confined by Two Horizontal Plates and Heated from Below', J. Heat Transfer (ASME) 81, 24-28.

Greenhut, G. K. and Khalsa, S. J. S.: 1987, 'Convective Elements in the Marine Atmospheric Boundary Layer. Part I: Conditional Sampling Statistics', J. Clim. Appl. Meteurol. 26, 813-822.

Haugen, D. A., Kaimal, J. C., and Bradley, E. F.: 1971, 'An Experimental Study of Reynolds Stress and Heat Flux in the Atmospheric Surface Layer', Quart. J. Roy. Meteorol. Soc. 97, 168-180.

Kaimal, J. C., Wyngaard, J. C., Haugen, D. A., Cote, O. R., Izumi, Y., Caughey, S. J., and Readings, C. J.: 1976, 'Turbulence Structure in the Convective Boundary Layer', J. Atmos. Sci. 33, 2152-2169.

Moeng, C.-H. and Wyngaard, J. C.: 1986, 'An Analysis of Closures for Pressure-Scalar Covariances in the Convective Boundary Layer', J. Atmos. Sci. 43, 2499-2513.

Monin, A. S. and Yaglom, A. M.: 1971, Statistical Fluid Mechanics: Mechanics of Turbulence, MIT Press, Cambridge, Mass., Vol. 1.

Monin, A. S. and Zilitinkevich, S. S.: 1969, 'On Description of Micro- and Meso-scale Phenomena in Numerical Models of the Atmosphere', WMO-IUGG Symposium on Numerical Weather Forecasting Tokyo, 26 Nov.-4 Dec. 1968, Techn. Rep. Japan Meteorol. Agency No. 67, I.105-I.121.

Panofsky, H. A. and Dutton, J. A.: 1984, Atmospheric Turbulence, J. Wiley, New York, 397 pp.

Paulson, C. A.: 1970, 'The Mathematical Representation of Wind Speed and Temperature Profiles in the Unstable Atmospheric Surface Layer', J. Appl. Meteorol. 9, 857-861. 
Prandtl, L.: 1932, 'Meteorologische Anwendung der Strömungslehre', Beitr. Phys. fr. Atmosph. 19, $188-202$.

Priestley, C. H. B.: 1954, 'Convection from a Large Horizontal Surface', Australian J. Phys. 7, 176-201.

Schmidt, H. and Schumann, U.: 1989, 'Coherent Structure of the Convective Boundary Layer Derived from Large-Eddy Simulations', DFVLR-IB-553-2/88, submitted to J. Fluid Mech.

Schumann, U., Hauf, T., Höller, H., Schmidt, H., and Volkert, H.: 1987, 'A Meso scale Model for the Simulation of Turbulence, Clouds and Flow over Mountains: Formulation and Validation Examples', Beitr. Phys. Atmosph. 60, 413-446.

Tennekes, H.: 1973, 'Similarity Laws and Scale Relations in Planetary Boundary Layers', in D. A. Haugen (ed.), Workshop on Micrometeorology, Amer. Meteor. Soc., Boston, pp. 177-216.

Townsend, A. A.: 1964, 'Natural Convection in Water over an Ice Surface', Quart. J. Roy. Meteorol. Soc. 90, 248-259.

Viswanadham, Y.: 1982, 'Examination of the Empirical Flux-Profile Models in the Atmospheric Surface Boundary Layer', Boundary-Layer Meteorol. 22, 61-77.

Wyngaard, J. C.: 1985, 'Structure of the Planetary Boundary Layer and Implications for its Modeling', J. Clim. Appl. Meteorol. 24, 1131-1142.

Wyngaard, J. C., Cote, O. R., and Izumi, Y.: 1971, 'Local Free Convection, Similarity, and the Budgets of Shear Stress and Heat Flux', J. Atmos. Sci. 28, 1171-1182.

Wier, M. and Römer, L.: 1987, 'Experimentelle Untersuchung von stabil und instabil geschichteten turbulenten Plattengrenzschichten mit Bodenrauhigkeit', Z. Flugwiss. Weltraumforsch. 11, 78-86.

Yaglom, A. M.: 1977, 'Comments on Wind and Temperature Flux-Profile Relationships', BoundaryLayer Meteorol. 11, 89-102. 